\title{
LA ESCUELA Y EL FORTALECIMIENTO COMUNITARIO
}

\author{
SCHOOL AND COMMUNITY EMPOWERMENT
}

\author{
Silvia López de Maturana Luna \\ Educadora de párvulos. \\ Doctora en Pedagogía \\ Académica de la Universidad de La Serena \\ Amunategui 851 \\ silvialml@gmail.com
}

Resumen: Este trabajo se focalizó en la escuela por considerar que puede potenciar las prácticas comunitarias. Al sistematizar las experiencias conocimos el alcance del trabajo pedagógico participativo de alumnos, profesores, familia y comunidad, al tiempo que reconstruimos el proceso gracias al cual fueron tomando conciencia sobre cómo utilizar los recursos naturales para ahorrar energía, cambiar el currículo escolar y cuidar del planeta.

Palabras Claves: Sistematización de experiencias, escuela y recursos naturales, fortalecimiento comunitario.

Abstract: The school and the community strengthening. This work focused in the school for thinking that it can promote the community practices. On having systematized the experiences, we knew the scope of the participative pedagogical work of students, teachers, family and community, at the time that we reconstruct the process thanks to which they were taking conscience on how using the natural resources to save energy, to change the school curriculum and to take care of the planet.

Key words: Systematization of experiences, school and natural resources, community strengthening. 


\section{INTRODUCCIÓN}

La escuela como eje vertebrador de experiencias transformadoras fortalece los aprendizajes no solo de los alumnos sino también de los actores sociales implicados en su formación. La experiencia de la escuela de Coquimbito $^{1}$ es un claro ejemplo de aquello por el involucramiento activo y permanente de toda la comunidad en el proyecto del uso de la energía solar ${ }^{2}$. El tema ambiental surgió de los mismos miembros de la comunidad quiénes necesitaban generar espacios de participación dentro de la escuela y abaratar costos de energía; una manera de hacerlo era unirse y trabajar por un mismo fin. Contaban con un Centro de Padres activo y con el clima adecuado, pero no sabían de la existencia de los hornos solares y de barro ni de cómo podían ahorrar energía.

La experiencia comunitaria comenzó con la llegada a la comunidad del director del proyecto medio ambiental ${ }^{3}$ quien después de realizar un diagnóstico del uso de los recursos naturales, las energías y los residuos sólidos de la comunidad de Coquimbito, presentó al director de la escuela la propuesta de construir hornos solares y de barro para el ahorro de la energía. Generó una serie de instrumentos metodológicos y de evaluación para implementar la temática ambiental que se refundieron en una matriz de integración al currículo escolar. Se instaló en la escuela y realizó talleres de capacitación a todo el personal y a los vecinos para sensibilizarlos en la temática ambiental, el diseño, construcción y uso de artefactos ecológicos de ahorro de energía ${ }^{4}$. Logró movilizar el entusiasmo, motivación, deseos y compromiso de participar en la mayoría de los miembros de la comunidad, favoreció la cohesión grupal y la interacción comunitaria.

La presidenta del Centro de Padres y la de la Junta de Vecinos tomaron el liderazgo en la comunidad escolar y vecinal reuniéndose periódicamente en la escuela. El trabajo colaborativo logró un cambio en sus estilos de vida y en la toma de conciencia sobre el deterioro del planeta y la importancia de la escuela como una institución que no sólo entrega contenidos. La activa participación de las mujeres fue fundamental para el cambio en la organización comunitaria quiénes empezaron a usar herramientas y tecnologías generalmente privativas de los hombres, lo que contribuyó a cambiar el rol de la mujer a través de la creación de espacios que les eran ajenos.

Se eligió sistematizar esa experiencia, por una parte, porque los actores sociales construyeron un sentido de comunidad que impactó en 
la transformación del estilo de vida a través de nuevas redes de colaboración entre vecinos y mostró la posibilidad de promover políticas sociales en educación. Por otra parte, para dar a conocer el enorme poder sistémico de la escuela que, si se lo propone, aglutina esfuerzos comunes a su alrededor.

La sistematización es un proceso de recuperación de una experiencia práctica formativa que permite comprender y explicar el sentido, fundamentos, lógicas y aspectos problemáticos de la experiencia "con el fin de transformar y cualificar la comprensión, experimentación y expresión de las propuestas educativas de carácter comunitario" (Ghiso, 2001). Es una propuesta metodológica cuyo profundo proceso de indagación y reflexión sobre la práctica, rescata la historia y le da nuevos significados, al tiempo que ofrece, recrea y crea conocimiento a partir de la práctica y de las relaciones que, en su tiempo, realizaron los actores sociales involucrados, razón por la cual implica un exhaustivo proceso de elaboración intelectual. Se presenta, generalmente, como una alternativa a la evaluación tradicionalmente aplicada a los proyectos sociales y educativos, generalmente presos del paradigma positivista que no muestran el sentido de los cambios y de la intervención social (Martinic, 1998).

Los sujetos de estudio fueron el director del colegio, profesorado, alumnado, apoderados, vecinos/as, la presidenta del Centro de padres del colegio y la de la Junta de Vecinos, con quienes se interactuó a través de entrevistas, filmaciones y conversaciones informales.

\section{PROBLEMA}

¿Cuáles son los dispositivos de participación utilizados por los miembros de la comunidad de Coquimbito que han hecho posible el fortalecimiento comunitario y el trabajo colaborativo en un proyecto medioambiental que favorece a toda la comunidad?

La comunidad necesitaba realizar acciones comunes para superar ciertos conflictos y problemas de convivencia entre vecinos, validar del rol de la escuela, fomentar el interés por sus actividades y aprovechar mejor las energías naturales ${ }^{5}$. Se construyó y cocinó en los hornos solares y de barro dentro de la escuela, lo que se transformó en una actividad social y educativa que congregó a las personas a su alrededor tal como la cocina lo es dentro del hogar. El proyecto contribuyó a bajar el nivel 
de los conflictos y a unir a la comunidad a través del trabajo participativo, donde era difícil que alguien se negara porque involucraba la calidad de la educación de sus hijos y la mejora de la gestión de la escuela.

Los profesores necesitaban asumir el compromiso con su entorno; conocer la cantidad y calidad de los recursos del medio; comprender conceptos como el ahorro de energía y utilización de tecnologías renovables; prever las consecuencias de las acciones propias para la recuperación y sustentabilidad del medio ambiente; y transferir esos aprendizajes de manera proactiva al alumnado. Los alumnos necesitaban aprender a conservar, recrear y expresar el patrimonio cultural y ambiental de sus comunidades, razón por la cual, debían estar preparados para revertir las condiciones deficitarias de su entorno y alcanzar un mejoramiento en su calidad de vida, y, en el mejor de los casos, estimulados a permanecer en su localidad y no ceder al proceso migratorio hacia las grandes ciudades (Corporación Héctor Barreto, donde pertenece el director del proyecto).

\section{ANTECEDENTES TEÓRICOS}

\section{Educación escolar, Participación, Medio ambiente y Sistematización.}

\section{Educación escolar}

La escuela es el ecosistema social por excelencia dada su potencialidad de agrupar y convocar a los diversos miembros de la comunidad, razón por la cual, si profesores y alumnos adoptan conductas sustentables para la protección del entorno y valoran los recursos del medio ambiente, pueden proyectarlos hacia el resto de la comunidad e instalar la conciencia de cambio sobre el tema medioambiental. Esa posibilidad educativa adquiere gran relevancia en las zonas rurales. Para la Corporación Héctor Barreto, proyectos de este tipo implican:

Una nueva forma de relacionarse con el entorno y de concebir el quehacer cotidiano, de aplicar el conocimiento y el uso de tecnología apropiada a sus condiciones geográficas, ambientales y culturales. Una forma concreta y creativa que pueda ser desarrollada en las aulas y en los otros espacios naturales de la escuela, transformando las tareas domésticas; cocer alimentos, hornear pan, calentar agua, utilizando tecnologías apropiadas y vinculándolas con los contenidos de sus 
asignaturas, y se constituyan en experiencias de aprendizaje significativas.

El riesgo de no encarnar las acciones educativas escolares en la vida cotidiana es la apatía y desvinculación, generalmente producidas por la separación que la escuela hace de su rol educador con el contexto social, cultural, económico y político en los cuáles está inmersa.

El tema conduce inevitablemente a debatir sobre qué es lo que interesa realmente en la educación escolar, ¿profundizar hacia la raíz de los problemas o quedarnos en la superficie programática?; ¿Cómo escapar de una racionalidad instrumental que en su deseo de eficacia nos aleja precisamente de las comprensiones más útiles para una buena discusión sobre la formación? (Martínez Bonafé, 2005, p. 2).

La educación es fundamental para despertar la conciencia hacia el cuidado del medio ambiente como uno de los grandes recursos que van quedando para ayudar a mejorar la calidad de vida del planeta. Esos son aspectos del diario vivir que, a pesar de su relevancia, permanecen ocultos u olvidados porque el mundo vive preso del inmediatismo y de las urgencias que dejan de lado los aspectos más fundamentales para la vida, y son algunas de las razones de por qué el tema educativo es el eje aglutinador de esta sistematización cuyo gran propósito fue contribuir a la mejora de la calidad de la educación integral del colegio de Coquimbito.

\section{Participación}

Lograr lo precedente necesita de la participación comunitaria entendida como un proceso reflexivo donde se hace, posee, transforma y se es, en un constante movimiento desde lo colectivo a lo individual y viceversa (Hernández, 1995, 1996, 1997 y Sánchez, 2000, en Montero, 2007, p. 226). La participación desde la perspectiva comunitaria implica el trabajo colectivo, aglutinador y relacional en constante transformación, donde se desarrolla la colaboración, el compromiso y la solidaridad en la comunidad y donde todos sus miembros, aportan, reciben, crean conocimiento y deciden libremente de acuerdo a sus derechos y deberes. Como tiene efectos concientizadores se generan pautas de acción que movilizan los recursos y crean otros gracias al desarrollo de la capacidad reflexiva y crítica en favor del fortalecimiento de la comunidad (Montero, 2007, p. 230). 
Podemos hablar de la relación entre sujeto y sujeto y la tensión dialéctica que supone su integración con la sociedad, razón por la cual, el acto de participar implica, entre sujeto y sujeto, romper voluntariamente, y a través de la experiencia, la relación asimétrica de sumisión y dependencia (Fals-Borda y Anisur Rahman, 1991, p. 10). Es relevante el concepto de participación al que aluden los autores recién señalados puesto que la conciben desde la historia real de la gente común, donde se toman en cuenta sus tradiciones, sentimientos y actitudes genuinas y democráticas. En este contexto el sujeto es considerado poseedor de experiencias y conocimientos válidos para no objetivarlo a través de estándares que terminan por anularlo, por lo tanto, es fundamental considerar el desarrollo de la autoestima positiva y de las buenas relaciones sociales, ya que son los soportes personales y sociales que toda persona necesita para su equilibrio bio-psico-social, desarrollo y bienestar (Cfr. Caron, 1996), y es uno de los principales dispositivos que permiten que la persona se sienta útil y necesaria a la sociedad.

Una de las maneras más efectivas de lograr una genuina participación es el aprendizaje gradual a través de la experiencia, que generalmente se adquiere a través de las vivencias en los grupos con trayectoria participativa. En caso contrario, se corre el riesgo de vivir experiencias acomodaticias, complacientes, rutinarias, superficiales y sin sentido de comunidad. En ese contexto, los miembros jóvenes de la organización participativa tienen muchas posibilidades de involucrarse de manera autónoma en la solución de los problemas de su comunidad mientras más pronto tengan posibilidades de formar parte de proceso de reflexión crítica y de toma de decisiones, ya que, de esa manera participarán y crearán dinámicas sociales que pueden transformar sus hábitos sociales.

Lo anterior se explica por el lugar que una persona ocupa en el proceso social y la autonomía que posee, lo que confluye para determinar sus percepciones, valoraciones y desarrollo personal. Por eso, los diferentes grados de proximidad y alejamiento espacio temporal, físico, intelectual y afectivo con que experimentamos la vida cotidiana dependen del grado de impacto que nuestras actividades tienen sobre nuestros intereses (Berger y Luckmann, 1999:40). El sujeto no es un ser aislado construido en solitario, sino que depende de una multiplicidad de factores complejos que le otorgan un puesto en el mundo en tanto creación, análisis, acatamiento, aislamiento o participación. Sin embargo, generalmente, se lo considera aislado asépticamente de sus 
respectivos entornos sociales donde pareciera que el individuo "fuera ontológicamente distinto a la multiplicidad de personas presentada como sociedad" (Elias, 2000:11). Una manera de revertir el aislamiento es promover la participación en experiencias conjuntas con el resto de la comunidad, por ejemplo, que los niños vean a sus padres y a sus profesores en otras actividades que no sean las habituales.

\section{Medio ambiente}

Para nadie es un misterio los efectos devastadores que el ser humano ha hecho al medio ambiente sin tomar conciencia de las consecuencias de los actos donde pareciera que se conoce el daño, pero nada se hace para evitarlo. Por ejemplo, la mayoría de los elementos de uso diario son desechables y lo que antaño se usaba por años hoy se usa y se tira, pues resulta más rentable fabricar un nuevo envase que reciclarlo. Eso hace que el planeta se esté llenando cada vez más de basura inservible que contamina permanentemente cada rincón de vida, así como el uso indiscriminado, entre otros, del agua, luz, calefacción y aire acondicionado. Cada vez somos más conscientes de que la supervivencia de la vida sobre la Tierra está en peligro y que, de una manera excesivamente alegre y poco previsora, estamos actuando de formas que originan problemas nuevos que podrían evitarse (Delval, 2006, p. 85).

La formación escolar es imprescindible para la toma de conciencia sobre el desarrollo sostenible, porque el futuro de los escolares se encuentra en las acciones cotidianas del presente y porque ellos pueden influir radicalmente en el desarrollo de la calidad de vida. Los niños y jóvenes tienen a su alcance las posibilidades de revertir los efectos del deterioro ambiental si en la escuela viven experiencias significativas y trascendentes.

Educar para la austeridad y no para el despilfarro es un gran desafío, sobre todo porque el discurso dominante de las empresas y de muchos medios de comunicación apunta a lo segundo, y porque sabemos que son muchos los escolares que adhieren a esas prácticas. Es necesario posibilitar a los estudiantes la construcción de nuevos significados para lo cual resulta imperativo que el profesorado se pregunte por lo que harán sus estudiantes una vez que adquieran el conocimiento. Eso, entre otros cuestionamientos, forma parte de la autonomía docente que puede 
posibilitar el desarrollo del proceso de aprendizaje significativo o puede reducirlo a un producto funcional y oportunista.

\section{Sistematización}

Lo precedente ameritó ser estudiado bajo la óptica de la sistematización, cuya perspectiva teórica la describe como humanitaria, democrática, comunitaria, y popular que busca transformaciones sociales estructurales. Eso conlleva el riesgo de ser interpretada de manera populista o que cualquiera hable de sistematizar las prácticas sin darle el valor profundo que conlleva (Cfr. Zúñiga, 1991, p. 2). Al generar nuevo conocimiento no transmite unilateralmente los conocimientos existentes ni repite linealmente lo dicho, sino que los relaciona, re-crea e interpreta.

Eso muestra una visión paradigmática diferente a la tradicional de la ciencia, puesto que se valoran y divulgan los saberes locales, sus expresiones y modos de conocer y comprender el mundo. Por lo tanto, se incluyen las emociones y la subjetividad que impulsa las prácticas de las personas (Jara, 2011, p. 5). Para Francke y Morgan (1995, p. 10), dado su carácter social, la sistematización consiste más en aprender de las prácticas que en elaborar conocimiento, sobre todo porque las personas beneficiarias son las reales protagonistas.

La génesis de la sistematización en América Latina se remonta a los trabajos de Educación Popular y a la Investigación Acción Participativa (IAP) donde se pretende mejorar la práctica a través de los conocimientos que se adquieren al investigarla y al evaluar sus fortalezas y debilidades. Todo eso favorece la interacción entre los actores sociales involucrados que trabajan, por un bien común, para tener una mayor comprensión de su propio trabajo y contribuir a los procesos de democratización y participación. No tiene un enfoque único ya que depende de los contextos y de los momentos históricos de cada comunidad. Tal como en la IAP, la sistematización pretende la producción y difusión del nuevo conocimiento y "acabar con el monopolio de la palabra escrita" (Fals- Borda y Anisur Rahman, 1991, p. 15).

No existe una definición consensuada sobre qué es la sistematización, lo que puede añadir confusión a la hora de entender el concepto, pero también nos abre puertas a nuestro propio aporte. El sentido democratizador de la sistematización de las prácticas implica dar 
la voz a las acciones para que se conozcan, respeten, afirmen y reivindiquen, por lo tanto, se trata de ofrecer los medios para buscar el sentido de las acciones y que puedan compartirse con los otros sujetos partícipes de dichas acciones (Cfr. Zúñiga, 1991, p. 2).

\section{METODOLOGÍA}

En consecuencia, la metodología utilizada es la Sistematización de la Experiencia ${ }^{6}$ que permitió recuperar información relevante y generar conocimiento susceptible de ser transferido a otras realidades. Se describió, reflexionó, analizó y documentó la información recabada sobre las maneras a través de las cuáles los actores sociales de la comunidad se unieron en un trabajo participativo para lograr metas comunes. Se realizó la interpretación crítica de la experiencia ya que gracias a su reconstrucción permitió descubrir y/o explicar la lógica del proceso llevado a cabo, los factores que intervinieron, cómo se relacionaron entre sí y por qué lo hicieron de esa manera. De modo que es muy distinto entenderla como sistematización de datos o información que como sistematización de experiencias (Cfr. Jara, 2001).

Implicó el análisis permanente de los diversos actores sociales que participaron en ella para descubrir cotidianamente nuevas realidades. Eso significó desprenderse de prejuicios para no caer, como señala Freire (n.d), en la tentación de lo obvio y evidente, que por lo mismo no se considera. Se evaluó cada parte del proceso lo que significó explorar sistemáticamente cada situación para darle sentido y, en el mejor de los casos, trascendencia. Hasta el más mínimo detalle tuvo relevancia para los análisis, aunque, finalmente, no todos se transformaron en información relevante, puesto que hubo que filtrar y seleccionar. En el proceso de reconstrucción de lo acontecido, lo que interesó fue realizar una interpretación crítica más que sólo reconstruirlo, de esa manera, fue posible extraer aprendizajes significativos para posibles acciones a futuro, mejorar nuestra propia práctica, compartir los hallazgos y contribuir al mejoramiento de la teoría (Jara, 2001).

Como la sistematización de experiencias se enmarca dentro del concepto de investigación colectiva de la IAP, recolectamos y sistematizamos la información gracias al aporte de los conocimientos de los mismos miembros de la comunidad a través de entrevistas en profundidad, otras semiestructuradas y más informales; filmaciones de 
conversaciones con los actores sociales; reuniones de pequeño y gran grupo con apoderados, alumnos, profesores y vecinos; observación participante en visitas al colegio, almacenes del barrio y casas de las familias para conocer los hornos y saber de su uso.

El proceso implicó dar cuenta de algo más que una cronología de los hechos y nos facilitó la descripción de lo sucedido. Esas son prácticas de la IAP que validaron un método participativo y dialógico que:

Provee una validación social de los conocimientos objetivos que no pueden ser adquiridos por otros medios individuales basados en trabajos de campo y encuesta. De esta manera, la confirmación se obtiene de los valores positivos del diálogo, de la discusión, de la argumentación o del consenso dentro de la investigación objetiva de las realidades sociales (Fals-Borda y Anisur Rahman, 1991, p. 14).

Recopilamos la mayor cantidad de datos y establecimos diversas relaciones con los actores sociales para recuperar lo vivido y lo alcanzado a través de la descripción de la experiencia, tomando en cuenta que el proceso de sistematización "exige descomponer y recomponer los elementos de la práctica y sus relaciones para comprender la totalidad de la experiencia" (Ruiz Botero, 2001, p. 7).

El diseño de la sistematización contempló tres fases: Inicio, Desarrollo y Final. La fase de Inicio, como punto de partida, correspondió a la delimitación precisa de la experiencia y partió con las preguntas iniciales: ¿por qué? ¿para qué? ¿cómo vamos a sistematizar? La fase de Desarrollo fue la recuperación de lo vivido y alcanzado, donde se describió la experiencia, se recuperó el proceso vivido y se ordenó y clasificó la información en categorías. Y en la fase Final se realizó un análisis crítico: ¿por qué pasó lo que pasó? Se obtuvieron conclusiones teóricas y prácticas y se comunicaron los aprendizajes.

\section{RESULTADOS Y ANÁLISIS}

Fase de Inicio: el proyecto comunitario comenzó en Marzo del 2008 y su implementación duró un año. Se sistematizó el 2010 donde se determinó el proceso e identificó a los participantes, los recursos disponibles, plazos, y la información con la que se contaba. Sabíamos que el proyecto solucionó la falta de trabajo colaborativo y la necesidad 
de participación y relación más profunda del colegio con la comunidad y con sus demandas.

Fase de Desarrollo: gracias a la recuperación del proceso vivido observamos que el proyecto fue útil a los vecinos para mejorar la calidad de vida, colaborar con el cuidado del medio ambiente y participar en un trabajo colaborativo con el resto de la comunidad; a las familias, para mejorar la convivencia escolar en los contextos privados y públicos; a la escuela para fortalecer la comunidad educativa escolar gracias a la participación y compromiso de sus actores y a la integración del cuidado medioambiental; al profesorado para establecer relaciones curriculares distintas a las tradicionales e inculcar la conciencia ecológica en los alumnos; a los apoderados, para fortalecer su participación en la escuela, generar redes de cooperación con sus hijos y profesores y comprender mejor las actividades curriculares; al alumnado, para formar parte de una comunidad más preocupada del medio ambiente, tomar conciencia de otras alternativas de ahorro de la energía y crear nuevas conversaciones en clases.

Fase Final: la experiencia en la comunidad impactó en la toma de conciencia de las consecuencias de las acciones conjuntas, gratificación de la calidad de vida, valor e interés de los vecinos por lo que sucede en la escuela, ahorro de energía y abastecimiento de nuevas tecnologías para el cuidado del medio ambiente.

\section{Participación comunitaria y educación escolar}

La participación comunitaria se observó en la producción e intercambio de conocimientos entre las familias a través de consejos, recursos y nuevas estrategias de producción. El proyecto le sirvió a la escuela, por una parte, para fortalecer el trabajo de la comunidad educativa escolar gracias a la participación y compromiso mutuo entre profesores, alumnos, padres y directivos y para integrar el currículo en sus aspectos más formales, puesto que el cuidado medioambiental fue un eje transversal. Por otra parte, para crear tiempos y espacios de socialización para la convivencia, intercambio libre de ideas, mejora de los procesos de socialización, y reforzamiento de las relaciones horizontales de trabajo colaborativo; por ejemplo, profesores, alumnos y apoderados construyendo un horno y cocinando juntos. 
El proyecto le sirvió al profesorado para establecer relaciones distintas a las tradicionales, lo que favoreció el clima emocional y organizacional del aula y para inculcar la conciencia ecológica en los alumnos. Los profesores construyeron un modelo educativo en la escuela que incorporó tecnologías ambientalmente apropiadas para el uso de la energía solar y el reciclaje de la materia orgánica. Señalaron que la experiencia fue muy enriquecedora para el currículo porque pudieron aplicar la enseñanza en relación al medio ambiente y aprendieron a cuidarlo. Destacaron y valoraron que esa experiencia fue y es una manera de involucrar el futuro de sus alumnos para que puedan obtener de los medios otros recursos para cuidar su entorno.

Me gustó mucho que todos los niños en la mayoría de los cursos, incluso los chiquititos, tenían un espacio para intervenir en la confección [de los hornos], desde ahí partíamos. Incluso haciendo al principio unos bosquejos, confeccionando en miniatura, en la asignatura de tecnología, los mismos artefactos que se hicieron después (profesora A).

Los profesores dieron cuenta del cambio en sus alumnos al incorporar el uso de los hornos en las planificaciones dentro del currículo escolar formal, y del propio cambio al modificar el uso de los libros de textos y crear los suyos, aun reconociendo lo mucho que les falta por aprender.

Con un curso hice una receta para enseñarles muchas estrategias de cómo cocinar y la limpieza que tienen que tener. Hicimos una actividad con los textos normativos relacionada con los choclos. Partimos desde la mazamorra del choclo, choclo cocido, pastel de choclo, humitas y pastelera, todo lo habido y por haber que se pueda hacer con choclo lo hicimos en octavo. La clase estuvo muy motivada. (Profesora C).

En las clases hubo nuevas conversaciones donde los alumnos relataban lo que sus madres hacían en los hornos que habían construido en sus casas, por lo cual, las actividades fueron más incentivadoras, innovadoras y creativas. Los alumnos se movilizaban llevando recetas de la escuela al hogar y viceversa, lo que alteró favorablemente la dinámica de la clase, porque los profesores debieron innovar en sus clases tradicionales. A un alumno de 10 años le llamó la atención ver que las madres vendían en el colegio los almuerzos cocinados en los hornos 
y la participación de casi todos los cursos y de los profesores. Otro alumno de 11 años señaló que

Es divertido, porque tuvimos que construir con las propias manos. Trabajo con mi mamá. Primero aprendimos con la señorita [profesora] y después yo me interesé y le empecé a ayudar a mi mamá. En la casa tenemos un horno, se ahorra gas y electricidad". Otra alumna dijo: "había un hoyo y sacamos tierra, le echamos agua después le echaron barro a los hornos. En la casa, atrás hay uno, lo construyó mi papito... sirven para no contaminar el ambiente.

La experiencia escolar reforzó el trabajo realizado en el hogar, tal como lo señaló otro alumno de 11 años: "aprendí en la escuela que no hay que contaminar el ambiente, allá atrás [patio de la escuela] tenemos un poquito de pasto, lo mojamos y después lo cubrimos para que se abrigue y después vaciamos la tierra para las plantas". Agregó que "ibamos a buscar piedras con carretillas por toda la escuela, lo hacíamos en las horas de clases". Los niños disfrutaron con esa experiencia de manera lúdica y significativa, lograron establecer relaciones más cercanas con sus profesores y familia y tomaron más conciencia del ahorro de energía ya que, según sus profesores, están preocupados de cuidar las luces y el agua.

\section{Categorías emergentes}

Las categorías que emergieron de la sistematización son el Liderazgo compartido, las Relaciones comunitarias, la Participación comunitaria y el Fortalecimiento comunitario. El liderazgo compartido entendido como el estilo de dirección consensuado y acordado entre los miembros de una comunidad se observó en la relación entre la presidenta de la Junta de Vecinos y la presidenta del Centro de Apoderados quiénes se conjuntaron para propiciar las reuniones y convocar a los apoderados para que participaran en las reuniones y en la construcción de los hornos.

Las Relaciones Comunitarias se observaron en el trabajo conjunto y voluntario de todos los participantes con la comunidad "promoviendo el desarrollo de proyectos de beneficio común" gracias a la concepción comunitaria de liderazgo (Cfr. Arango, 2008:344). El liderazgo de las mujeres del proyecto creó un clima de convivencia positiva en la organización comunitaria, gracias al entusiasmo en el 
trabajo, la elaboración de productos que reportaron beneficios a todos, y la posibilidad de crear pequeñas empresas de venta de pan a bajo costo energético.

La Participación Comunitaria, fue el mecanismo que permitió hacer realidad el derecho de las personas a ser sujetos de los procesos específicos que cada grupo deseaba para mejorar la vida de su comunidad, "no sólo representa una posibilidad en sí misma para tomar decisiones que afectan a su vida colectiva, sino que constituye el mecanismo que permite hacer realidad el derecho de todas las personas a ser sujetos de su historia, es decir, sujetos de los procesos específicos que cada grupo desea para mejorar la vida de su comunidad" (Musitu, 2004, p. 5).

Y el Fortalecimiento Comunitario se observó en la dinámica social que logró movilizar y utilizar los recursos. El director del proyecto, el director del colegio y las presidentas del Centro de Padres y de la Junta de Vecinos, respectivamente, formaron parte del proceso interno que logró aglutinar la participación de la mayoría de los miembros de la comunidad a través de un trabajo constante y sostenido. Por ejemplo, en los talleres y sesiones de sensibilización ecológica, construcción de materiales y de retroalimentación; en las modificaciones curriculares de los profesores; en las dinámicas distintas para dar una clase, donde intencionalmente se utilizaron los recursos que se estaban trabajando en el proyecto; en la utilización de los hornos para que las madres apoyaran con los ingresos al hogar, que mayoritariamente estaba a cargo de los hombres; y los tiempos y espacios para la participación activa y organizada de la comunidad.

\section{¿Por qué pasó lo que pasó?}

La experiencia de sistematización nos permitió conocer las maneras a través de las cuáles es posible fortalecer y cohesionar a una comunidad a pesar de las dinámicas relacionales de cualquier grupo humano diverso. Eso puede subsanarse, como en este caso, en torno a una experiencia que redunda en gratificaciones para su calidad de vida, a través del liderazgo apreciativo y distribuido, la clara definición de roles, y las fortalezas o aciertos desarrolladas en el proyecto de construcción de los hornos. Eso nos lleva a reflexionar sobre las posibilidades de extrapolación a otras comunidades gracias a las 
estrategias conocidas en esta comunidad y posibilitar una transformación en la vida comunitaria.

El liderazgo de las mujeres fue fundamental para los logros de esa experiencia, sobre todo en un contexto en el cual las dos mujeres líderes tuvieron un rol aglutinador de apoderados y de vecinos. La potenciación de su rol fue un elemento fundamental por la mutua convicción de la fortaleza del proyecto y de la necesidad de reforzar la participación comunitaria dentro de la escuela, favorecer la economía de los hogares y mejorar la calidad de vida del entorno. Su liderazgo favoreció el cambio del rol de la mujer de sujeto pasiva a sujeto activa y productora de sus propios recursos.

La lógica interna que se desarrolló en la comunidad fue cambiando progresivamente desde asistir a los talleres para aprender sobre energías renovables y construcción de los hornos, hasta tomar conciencia de la relevancia del trabajo conjunto de colaboración y organización para llevar adelante el proyecto con los logros de participación alcanzados. Aprendieron a participar haciéndolo, y ese proceso implicó la utilización intencionada de los recursos disponibles y la toma de conciencia de cada una de las acciones realizadas para su logro.

El fortalecimiento comunitario se observó en la colaboración entre los diferentes miembros de la comunidad cuyas consecuencias los beneficiaron en conjunto. Eso hizo posible procesos de participación entre personas que antes solo tenían relaciones formales, sobre todo en el colegio: profesor-alumno; profesor-apoderado; profesor-director; profesores-profesores; alumnos- alumnos.

En efecto, si las personas de una comunidad no comprenden ni han pensado en la posibilidad de llevar a cabo una determinada acción, ni tienen alguna forma de organización o de solidaridad, lo primero que se debería hacer es desarrollar esas condiciones internas mínimas (Montero, 2003, p. 14).

\section{Parámetros de impacto}

Introducción de la temática ambiental y la utilización de los hornos dentro del currículo; Procesos de convivencia distintos a los tradicionales; Prácticas sociales de los profesores/as con liderazgos 
democráticos que no se imponen; Madres que ocuparon los espacios escolares para algo más que ir a dejar o a buscar a sus hijos; Escuela como eje potenciador; Tránsito desde una pedagogía burocrática a una pedagogía más crítica y reflexiva de su entorno.

\section{CONCLUSIONES}

... es necesario que cada barrio o cada comuna cuente con una organización comunitaria o una institución que garantice el desarrollo de proyectos culturales que animen la vida comunitaria y el fortalecimiento del sentido de pertenencia al barrio o a la comuna (Arango, 2008, p. 374).

\section{Liderazgo y gestión colaborativa}

Se fortaleció el liderazgo colaborativo y se abrieron tiempos y espacios para la emergencia de otros liderazgos que asumieran tareas de responsabilidad creciente si el caso lo requería, por ejemplo, hacerse cargo de la coordinación de los talleres, organización de los materiales para la construcción de los hornos, preparación de los alimentos y presentación de los platos preparados a la comunidad. Supieron gestionar con el director del proyecto y con sus pares los recursos necesarios para movilizarlos hacia el logro común. Uno de los mejores indicadores de la comunidad potenciadora fue compartir las responsabilidades activas en los procesos de definición de los objetivos, toma de decisiones, resolución de los problemas, y en el apoyo a los acuerdos aprobados (Musitu, 2004, p. 14).

\section{Identidad comunitaria y experiencias de participación}

Los diferentes miembros de la comunidad dieron cuenta de la identidad comunitaria a través de la colaboración, apoyo, contento, entusiasmo, compromiso y sentido de comunidad al establecer relaciones de proximidad afectiva, seguridad y estabilidad percibida entre los miembros que comparten una historia común (Cfr. Puddifoot, 2003:88, en Montero, 2007, p. 219). "Saberse y sentirse miembro de una comunidad, o si se prefiere, de una organización o simplemente de un 
grupo, tiene, como muestran numerosas investigaciones, unos efectos positivos sobre el bienestar de la persona" (Musitu, 2004, p. 12).

Los padres y profesores concordaron que el logro del fortalecimiento de la comunidad y la construcción del sentido de identidad comunitaria se produjo por las oportunidades que los niños y jóvenes tuvieron de vivir experiencias de participación. Creemos que la trayectoria participativa sostenida en el tiempo puede potenciar y hacer perdurables esos procesos.

\section{Fortalecimiento comunitario y establecimiento de redes}

La comunidad se fortaleció, por una parte, porque sus miembros desarrollaron conjuntamente capacidades y recursos para lograr la transformación de su entorno según sus necesidades y aspiraciones, lo que implicó la transformación de sí mismos (Montero, 2003); y, por otra, porque tuvieron oportunidades de participación, pusieron en juego la motivación y los recursos para realizar acciones para mejorar la vida de la comunidad, desarrollaron estrategias para resolver sus problemas e hicieron esfuerzos por mejorar su calidad de vida (Musitu, 2004, p.2). Los recursos más utilizados fueron las redes asociativas cohesionadas entre sí y receptivas a las necesidades y demandas de los miembros de la comunidad, que permitieron que los involucrados en el proyecto supieran lo que tenían, lo que querían y que podían hacerlo en un marco de valores ecológicos compartidos.

\section{Compromiso ético y comunidad sustentable}

No solo desarrollaron paulatinamente la toma de conciencia de la necesidad de participación para el logro de los objetivos comunes, sino de la necesidad de cuidar el medio ambiente a partir del conocimiento y utilización de nuevas tecnologías para el aprovechamiento de los recursos naturales, lo que se unió al compromiso ético con la comunidad. Eso los llevó a involucrarse en acciones colectivas en beneficios de todos (Cfr. Montero, 2003, p. 6). Se observó una comunidad potenciadora caracterizada por un deseo de construir una comunidad sustentable que utilizó las energías del medio para su mejor desarrollo con valores de solidaridad, responsabilidad y colaboración entre sus miembros. Las actividades desarrolladas por los profesores mostraron una clara 
intención de cuidado del ambiente y toma de conciencia de que los recursos del medio no son inagotables.

\section{Aprendizajes para la escuela}

La motivación de los miembros de la comunidad para sostener el proyecto y realizar innovaciones se logró gracias a la participación conjunta de los diferentes estamentos escolares. El profesorado se dio cuenta de su posibilidad real de innovación dentro de las aulas escolares y que se puede aprender de maneras no tradicionales. Se abrieron posibilidades de re-formulación de políticas educacionales para movilizar nuevas formas de educación para la convivencia que revirtieron, de alguna manera, la tradición escolarizada producto de las relaciones verticales. Se tomó conciencia de que los niños necesitan aprender a participar e involucrarse, de manera autónoma, en acciones dirigidas a solucionar los problemas de su comunidad.

La escuela se valoró en cuanto a las posibilidades de propagar otros aprendizajes y las madres, especialmente, ocuparon los tiempos y espacios escolares para algo más que ir a dejar o a buscar a sus hijos. El trabajo de construcción de hornos y su utilización dentro de la escuela influyó en el compromiso de los profesores y de la familia con el proyecto educativo ambiental en detrimento del mero activismo escolar, lo que puede traer consecuencias trascendentes si es que se siguen potenciando esas acciones. Eso fue una de las grandes preocupaciones que impulsaron el desarrollo del proyecto en la escuela.

\section{Identificación con el proyecto}

El proceso de identificación con el proyecto por parte de los diferentes miembros de la comunidad escolar abrió posibilidades para transitar desde una pedagogía burocrática a una pedagogía más crítica y reflexiva de su entorno. La pedagogía crítica ${ }^{7}$ es marginal en el marco de la comunidad educativa en general (Gore, 1996, p. 30), razón por la que resultaron relevantes las relaciones educativas entre profesores, padres, madres, apoderados, y alumnos, para resituar el sentido de la acción educativa, y satisfacer, en este caso, la necesidad de participación ciudadana y de conciencia ecológica. 
La sistematización de este proyecto fue un proceso altamente participativo, porque nos identificamos con él y nos pusimos al servicio de la comunidad intentando reconstruir juntos el proceso. Desarrollamos el trabajo según lo establecido y retroalimentamos el proceso, establecimos en conjunto los objetivos y buscamos los mejores medios para lograrlos. Aprendimos de cada una de las acciones y pudimos dar forma a otras, que probablemente puedan realizarse a futuro.

\section{Notas:}

${ }^{1}$ Coquimbito, es un pueblo semi rural del mismo nombre, ubicado en el Valle de Elqui, distante a $15 \mathrm{Km}$. de La Serena.

${ }^{2}$ En el Valle de Elqui, existe una gran variedad de comunidades rurales que no siempre se vinculan con la institucionalidad pública (presentación de proyectos) quedando, por esto, relegados de los programas que mejoren el nivel de vida de la comunidad y su entorno. Producto de eso, en diversas localidades se mantienen antiguas prácticas asociadas al uso de la energía, dañinas para las personas y el medio ambiente. Por ejemplo, el mal uso de las técnicas de quemado de la leña con fines domésticos, que llegan a perder hasta el $80 \%$ del calor producido, contribuyendo a la deforestación, afectando recursos como el agua y el suelo, y comprometiendo la productividad de la agricultura (Cfr. Corporación Héctor Barreto).

${ }^{3}$ Rafael Bahamondes de la Corporación Héctor Barreto. Dicha Corporación capacita y promociona liderazgos ciudadanos, fortalecimiento del tercer sector y desarrollo tecnológico.

${ }^{4}$ En la mayoría de los sectores rurales de la Región de Coquimbo se mantienen antiguas prácticas asociadas al uso de la energía dañinas para las personas y el medio ambiente, razón por la cual, la sistematización puede ser un canal viable de información para que otras comunidades visualicen sus carencias, demandas y propuestas, quienes también podrán obtener asesoría para la formulación de proyectos, conocer las fuentes de financiamiento, descubrir sus potencialidades y aprender estrategias de participación.

${ }^{5} \mathrm{El}$ sol, inestimable recurso natural, al alcance diario, fue el incentivo natural para la construcción de los hornos solares y los alimentos que de ellos se obtiene. Eso implica el consumo de energía domiciliaria que 
satisface a menor costo económico y ambiental el desarrollo del sector rural, es una muestra evidente de la presencia del tema productivo dentro de los grandes temas ambientales.

${ }^{6}$ Las sugerencias metodológicas están basadas en el documento de Chávez, Jorge (2007). Ordenando y analizando. Una metodología para la sistematización. REDESA-CARE - Perú.

${ }^{7}$ Cuando nos referimos a pedagogía crítica entendemos la responsabilidad y compromiso de nuestras relaciones con el mundo reconociendo nuestra participación activa en la producción de conocimiento en sus dimensiones moral, política y cultural (Mac Laren, 1998 , p. 228). Significa pensar una nueva forma de hacer docencia, en donde las relaciones con los alumnos son más humanas y significativas $\mathrm{y}$ en donde propiciamos que profesores, alumnos, padres y madres asuman su responsabilidad de comprender el mundo para transformarlo.

\section{REFERENCIAS BIBLIOGRÁFICAS}

Arango, C., 2008

Psicología comunitaria de la convivencia. Cali, Colombia. Edita: U del Valle.

Berger, P., Luckmann, La construcción social de la realidad. T., 1999 Buenos Aires: Amorrortu editores.

Boletín de La naturaleza del proyecto de sistematización. 1997 sistematización: preguntas claves. Congreso de IAP en Cartagena de Indias, Colombia.

Caron, J., 1996

Una Teoría Ecológica para la Intervención Comunitaria: Acceso y Conservación de los Recursos. Revista Intervención Psicosocial, Vol. V, Nº14.

Chávez, J., 2007 Ordenando y analizando. Una metodología para la sistematización. Perú: REDESA - CARE.

Cuadernillo 1. Viendo la sistematización. Qué es la sistematización. www.alboan.org/

Delval, J., 2006 archivos/1viendo.pdf

Hacia una escuela ciudadana. Madrid: Morata. 
Documentos. 2008

Elias, N., 2000

Fals-Borda, O. y Acción y Conocimiento. Cómo romper Anisur Rah- man, M., el monopolio con Investigación - Acción 1991 Participativa. Bogotá: CINEP.

Francke, M. $\quad$ y La sistematización: apuesta por la Morgan, M.L., 1995 generación de conocimientos a partir de las experiencias de promoción. Materiales didácticos $\mathrm{N}^{\mathrm{o}}$ 1, Lima: Escuela para el Desarrollo.

Ghiso, A., 2001

Sistematización de experiencias en Educación popular. Memorias foro: los contextos actuales de la Educación Popular. Medellín en RUIZ BOTERO, L. La sistematización de prácticas. www. sistematización.

Gore, J., 1996

Controversias entre las pedagogías. Madrid: Morata.

Heller, A., 1996

Una revisión de la teoría de las necesidades. Barcelona: Paidós. - 1998. Sociología de la vida cotidiana. Barcelona: Península.

Jara, O., 2001

Presentación realizada en el mes de Abril 2001, Cochabamba, Bolivia, en el Seminario ASOCAM: Agricultura Sostenible Campesina de Montaña, organizado por Intercooperación.

López de Maturana, Los buenos profesores: educadores S., 2010 comprometidos con un proyecto educativo. La Serena: Editorial Universidad de La Serena.

Mc Laren, P., 1998 Pedagogía crítica y cultura depredadora. Barcelona: Paidós.

Martínez Bonafé, J., Enseñar en la Universidad Pública. 2011 Sujeto, Conocimiento y poder en la 
Martinic, S., 1998

Montero, M., 2007

Musitu, G., 2004

Zúñiga, R., 1991
Educación Superior. Valencia: Universitat de València. - 2005. La Formación del profesorado y el discurso de las competencias. XI Congreso Internacional de formación del profesorado: Europa y calidad docente ¿convergencia o reforma educativa? Segovia.

El objeto de la Sistematización y sus relaciones con la Evaluación y la Investigación. Ponencia presentada al Seminario latinoamericano: sistematización de prácticas de animación sociocultural y participación ciudadana en América Latina. Medellín, Fundación Universitaria Luis Amigó CEAAL.

Introducción a la psicología comunitaria. Desarrollo, conceptos y procesos. Buenos Aires: Paidós. - 2003. Cap. 2. El Fortalecimiento en la Comunidad en Teoría y Práctica de la Psicología Comunitaria. Buenos Aires: Paidós.

Cap. VI. Desarrollo Comunitario y Potenciación (Empowerment) en Introducción a la Psicología Comunitaria de Musitu, G.; Herrero J.; Cantera L.; Montenegro M. Barcelona: ED UOC.

Ruiz Botero, L., 2001 La sistematización de prácticas. Sistematización experiencia de convivencia Liceo Nacional Marco Fidel Suárez. Instituto Nacional de Capacitación IPC. Medellín, Colombia. Sobre el sistematizar. Trabajo Social, Santiago de Chile, n61. - 1984. Ponencia en Seminario de Talagante s/r. 